\title{
Pricing of American Put Option under a Jump Diffusion Process with Stochastic Volatility in an Incomplete Market
}

\author{
Shuang Li, ${ }^{1,2}$ Yanli Zhou, ${ }^{1,3}$ Xinfeng Ruan, ${ }^{2}$ and B. Wiwatanapataphee ${ }^{4}$ \\ ${ }^{1}$ Department of Maths and Statistics, Curtin University, Perth, WA 6845, Australia \\ ${ }^{2}$ School of Economic Mathematics, Southwestern University of Finance and Economics, Chengdu 611130, China \\ ${ }^{3}$ School of Finance, Zhongnan University of Economics and Law, Wuhan 430073, China \\ ${ }^{4}$ Department of Mathematics, Faculty of Science, Mahidol University, Bangkok 10400, Thailand
}

Correspondence should be addressed to Yanli Zhou; ylzhou8507@gmail.com

Received 29 December 2013; Accepted 17 January 2014; Published 26 February 2014

Academic Editor: Yonghong Wu

Copyright (C) 2014 Shuang Li et al. This is an open access article distributed under the Creative Commons Attribution License, which permits unrestricted use, distribution, and reproduction in any medium, provided the original work is properly cited.

\begin{abstract}
We study the pricing of American options in an incomplete market in which the dynamics of the underlying risky asset is driven by a jump diffusion process with stochastic volatility. By employing a risk-minimization criterion, we obtain the Radon-Nikodym derivative for the minimal martingale measure and consequently a linear complementarity problem (LCP) for American option price. An iterative method is then established to solve the LCP problem for American put option price. Our numerical results show that the model and numerical scheme are robust in capturing the feature of incomplete finance market, particularly the influence of market volatility on the price of American options.
\end{abstract}

\section{Introduction}

Since the pioneering work of Black-Scholes [1] and Merton [2] on the pricing of options, option pricing has become one of the predominant concerns in financial market. By relaxing some of the restrictive assumptions, such as the assumption of constant volatility and the geometric Brownian motion for the price of the underlying asset, many option pricing models have been proposed. Today, an increasing amount of literature has focused on stochastic volatility models (see, e.g., [3]), jump diffusion or Lévy process models $[4,5]$, which fit some features of the observed empirical market data. In this paper, in constructing our price model, we will take account of both the feature of stochastic volatility as in Heston's model and the feature of jump diffusion as in Bates [6]. The combination of the stochastic volatility and jump diffusion in the same model enables the model to capture the nature of the real financial market and yields a challenging and difficult mathematical problem for analysis.

Because of infrequent trading or by the presence of additional sources of uncertainty, the completeness of the market often breaks down. Thus, replicating the payoff of a contingent claim by a portfolio is possible, and various equivalent martingale measures (EMM) have been adopted. The question is that, among the equivalent martingale measures, one needs to establish certain criterions to determine the appropriate measure to be adopted. A unique equivalent martingale measure by using utility maximization has been identified by many authors including Kallsen [7], Bielecki and Jeanblanc [8], and Cvitanić et al. [9]. It was also found that, under the minimal martingale measure, there exists a unique risk-minimizing strategy hedging of contingent claims in complete market [10]. The criterion under the minimal martingale measure is thus referred to as riskminimization criterion (see [11]). According to this, it is possible to price option with risk-minimization criterion. In this paper, we use the minimal martingale measure and consider the switch of assets prices with jump diffusion and stochastic volatility and obtain the Radon-Nikodym derivative and a linear complementarity problem for the pricing of American option.

Unlike European options, American options, which can be exercised any time before the expiring date, have to be priced usually numerically. Comparing to European options 
which lead to a partial integrodifferential equation, American options lead to inequalities in the form of a linear complementarity problem (LCP). In the American option pricing context, several methods have been proposed to approximate the linear complementarity problems resulting from American option pricing. These include the finite difference method, the operator splitting method, the multigrid method, and the penalty method [12]. Moreover, Tavella and Randall [13] described a stationary iterative method for pricing European options, and then Salmi and Toivanen [14] proposed a generalization of this iterative method to price American option. But they did not consider any multifactor jump diffusion models such as stochastic volatility models with jumps. Our paper aims to further develop previous numerical method to deal with multifactor jump diffusion models via the employment of the risk-minimization criterion to obtain the Radon-Nikodym derivative for the minimal martingale measure.

The rest of the paper is organized as follows. In Section 2, we discuss the pricing model with stochastic volatility and jump diffusion under the minimal martingale measure and then derive the linear complementarity problem under the risk-minimization criterion. Section 3 presents the discrete space and time approximation for the LCP problem. The method of projected successive overrelaxation (projected SOR) is used to yield our results. Numerical results and discussion are reported in Section 4.

\section{The Model under Minimal Martingale Measure}

Taking into account the jumps in the evolution process of the underlying asset, we introduce the following stochastic volatility (1) based on the work in Heston [3]:

$$
\begin{gathered}
\frac{d X_{t}}{X_{t^{-}}}=\mu d t+\sqrt{V_{t}} d W_{t}^{1}+\int_{R}(y-1) \widetilde{N}(d t, d y) \\
d V_{t}=\alpha\left(\varphi-V_{t}\right) d t+\sigma \sqrt{V_{t}} d W_{t}^{2}
\end{gathered}
$$

where $W_{t}=\left(W_{t}^{1}, W_{t}^{2}\right)$ is defined on the filtered complete space $\left(\Omega, \mathscr{F},\left\{\mathscr{F}_{t}\right\}_{t \geq 0}, P\right)$ with 2-dimensional standard Brownian motion with correlation coefficient $d W_{t}^{1} d W_{t}^{2}=\rho d t, \rho \in$ $[-1,1]$. The asset price has drift $\mu$ and $\bar{N}(d t, d y)=N(d t, d y)-$ $v(d y) d t$ is the compensated jump measure, $N(d t, d y)$ denotes the jump measure, and $v(d y)$ is the Lévy measure, $\sigma$ governs the volatility of the variance process, and $\alpha$ and $\varphi$ are the mean reversion coefficients to ensure that the variance will drift back to $\varphi$ at the rate of $\alpha$.

According to the discussions in the reference by Föllmer and Schweizer [10], the optimal hedging strategy may be established with the minimal martingale measure and it has been proved that the minimal martingale measure can be determined uniquely. Using Theorem 3.1 in Heston [3], the Radon-Nikodym derivative under the minimal martingale measure $q$ is

$$
\begin{aligned}
Z_{t}=\exp (- & \int_{0}^{t} \theta_{u} \sqrt{V_{u}} d W_{u}^{1}-\frac{1}{2} \int_{0}^{t} \theta_{u}^{2} V_{u} d u \\
& +\int_{0}^{t} \int_{R} \ln \left(1-\theta_{u}(y-1)\right) N(d u, d y) \\
& \left.+\int_{0}^{t} \int_{R} \theta_{u}(y-1) v(d y) d u\right)
\end{aligned}
$$

where

$$
\theta_{u}=\frac{\mu_{u}-r_{u}}{V_{u}+\int_{R}(y-1)^{2} v(d y)}
$$

and $r_{t}$ is the risk-free rate of interest in the discounted risky asset price process $\widehat{X}_{t}=e^{-\int_{0}^{t} r_{u} d u} X_{t}$. The derivation of (2) is given in Appendix A. Correspondingly, the Brownian motion and the compensated Poisson process under the minimal martingale measure are derived as follows:

$$
\begin{gathered}
\widetilde{W}_{t}^{1}=W_{t}^{1}+\int_{0}^{t} \theta_{u} \sqrt{V_{u}} d u \\
\widetilde{W}_{t}^{2}=W_{t}^{2}+\rho \int_{0}^{t} \theta_{u} \sqrt{V_{u}} d u \\
\widetilde{v}(d y) d t=\left(1-\theta_{u}(y-1)\right) v(d y) d t \\
\widetilde{N}(d t, d y)=N(d t, d y)-\widetilde{v}(d y) d t
\end{gathered}
$$

In our model, there are two dimensions of risks from the Lévy process and the Brownian motion. Thus the financial market is not complete and such a claim has an intrinsic risk. In this context, we construct the pricing model under the riskminimization criterion by employing the minimal martingale measure method, and therefore model (1) can be written as follows:

$$
\begin{gathered}
\frac{d X_{t}}{X_{t^{-}}}=\mu d t+\sqrt{V_{t}} d \widetilde{W}_{t}^{1} \\
+\int_{R}(y-1) \widetilde{N}(d t, d y)-\theta_{t} V_{t} d t \\
\quad-\int_{R} \theta_{t}(y-1)^{2} v(d y) d t \\
d V_{t}=\alpha\left(\varphi-V_{t}\right) d t+\sigma \sqrt{V_{t}} d \widetilde{W}_{t}^{2}-\rho \sigma \theta_{t} V_{t} d t
\end{gathered}
$$

The value of the American put option $P\left(t, X_{t}, V_{t}\right)$ at time $t$, under strike price $K$ and maturity date $T$, is

$$
P\left(t, X_{t}, V_{t}\right)=\max _{\tau} \mathbf{E}^{q}\left[e^{-\int_{t}^{\tau} r_{u} d u}\left(K-X_{\tau}\right)^{+} \mid F_{t}\right]
$$

where $\tau$, representing the stopping times in $[0, T]$, is to be determined to maximize the discounted option payoff. 
Under the minimal martingale measure $q$, we obtain the following partial integrodifferential equation (see Appendix B for the detailed derivation):

$$
\begin{aligned}
\frac{\partial P}{\partial t}= & \left(r+K_{0}-\theta_{t} K_{1}\right) P(t, X, V)-\frac{\partial P}{\partial X}\left(r-K_{1}+\theta_{t} K_{2}\right) X \\
& -\frac{\partial P}{\partial V}\left(\alpha(\varphi-V)-\rho \sigma \theta_{t} V\right)-\frac{1}{2} \frac{\partial^{2} P}{\partial X^{2}} V X^{2} \\
& -\frac{\partial^{2} P}{\partial X \partial V} X V \sigma \rho-\frac{1}{2} \frac{\partial^{2} P}{\partial V^{2}} V \sigma^{2} \\
& -\int_{R} P(t, X y, V) v(d y) \\
& +\theta_{t} \int_{R} P(t, X y, V)(y-1) v(d y)
\end{aligned}
$$

where

$$
\begin{gathered}
K_{0}=\int_{R} v(d y), \\
K_{1}=\int_{R}(y-1) v(d y), \\
K_{2}=\int_{R}(y-1)^{2} v(d y) .
\end{gathered}
$$

Under Merton's jump diffusion model, we assume $v(d y)=$ $\lambda f(y) d y$, where $\lambda$ is the jump intensity and $f(y)$ is distribution of the jump size $y$, and assume that the stochastic volatility $V$ is the log-normal distribution with the density

$$
f(y)=\frac{1}{y \delta \sqrt{2 \pi}} \exp \left(-\frac{(\log y-\gamma)^{2}}{2 \delta^{2}}\right) .
$$

Let $\lambda=1$; thus the notations under the log-normal density functions above are $K_{0}=1, K_{1}=e^{\delta / 2+\gamma}-1$, and $K_{2}=(1 / \sqrt{1-}$ $\left.2 \delta^{2}\right) e^{\gamma^{2} /\left(1-2 \delta^{2}\right)}-2 e^{\delta / 2+\gamma}+1$.

Now we take the time transform $\tau=T-t$ and introduce an operator $L$ by

$$
\begin{aligned}
L \widetilde{P}= & -\left(r+K_{0}-\theta K_{1}\right) \widetilde{P}(\tau, X, V)+\frac{\partial \widetilde{P}}{\partial X}\left(r-K_{1}+\theta K_{2}\right) X \\
& +\frac{\partial \widetilde{P}}{\partial V}(\alpha(\varphi-V)+\rho \sigma \theta V)+\frac{1}{2} \frac{\partial^{2} \widetilde{P}}{\partial X^{2}} V X^{2} \\
& +\frac{\partial^{2} \widetilde{P}}{\partial X \partial V} X V \sigma \rho+\frac{1}{2} \frac{\partial^{2} \widetilde{P}}{\partial V^{2}} V \sigma^{2} \\
& +\int_{R} \widetilde{P}(\tau, X y, V) v(d y) \\
& -\theta \int_{R} \widetilde{P}(\tau, X y, V)(y-1) v(d y) .
\end{aligned}
$$

Then, the value of the American put option satisfies the following linear complementarity problem:

$$
\begin{aligned}
\text { LCP: } & \frac{\partial \widetilde{P}}{\partial \tau}-L \widetilde{P}_{\tau} \geq 0, \quad \widetilde{P}_{\tau} \geq\left(K-X_{\tau}\right)^{+} \\
& \left(\frac{\partial \widetilde{P}}{\partial \tau}-L \widetilde{P}_{\tau}\right)\left[\widetilde{P}_{\tau}-\left(K-X_{\tau}\right)^{+}\right]=0
\end{aligned}
$$

with the boundary conditions

$$
\begin{gathered}
\widetilde{P}(\tau, 0, V)=K, \\
\frac{\partial \widetilde{P}(\tau, \bar{X}, V)}{\partial X}=0, \\
\widetilde{P}(\tau, X, 0)=(K-X)^{+}, \\
\frac{\partial \widetilde{P}(\tau, X, \bar{V})}{\partial V}=0
\end{gathered}
$$

and the initial condition

$$
\widetilde{P}(0, X, V)=(K-X)^{+} .
$$

\section{Numerical Discretization}

We use the uniform grid $\Delta \tau=\Gamma / n, \Delta x=\bar{X} / m$, and $\Delta v=\bar{V} / l$ on the domain $[0, \Gamma] \times[0, \bar{X}] \times[0, \bar{V}]$. Denoting $\widetilde{P}_{i, j}=\widetilde{P}(\tau, i \Delta x, j \Delta v)$ and using the central difference scheme, we have

$$
\begin{gathered}
\frac{\partial \widetilde{P}}{\partial x}\left(\tau, x_{i}, v_{j}\right)=\frac{\widetilde{P}_{i+1, j}(\tau)-\widetilde{P}_{i-1, j}(\tau)}{2 \Delta x}, \\
\frac{\partial^{2} \widetilde{P}}{\partial x^{2}}\left(\tau, x_{i}, v_{j}\right)=\frac{\widetilde{P}_{i+1, j}(\tau)-2 \widetilde{P}_{i, j}(\tau)+\widetilde{P}_{i-1, j}(\tau)}{(\Delta x)^{2}}, \\
\frac{\partial \widetilde{P}}{\partial v}\left(\tau, x_{i}, v_{j}\right)=\frac{\widetilde{P}_{i, j+1}(\tau)-\widetilde{P}_{i, j-1}(\tau)}{2 \Delta v}, \\
\frac{\partial^{2} \widetilde{P}}{\partial v^{2}}\left(\tau, x_{i}, v_{j}\right)=\frac{\widetilde{P}_{i, j+1}(\tau)-2 \widetilde{P}_{i, j}(\tau)+\widetilde{P}_{i, j-1}(\tau)}{(\Delta v)^{2}}, \\
\frac{\partial^{2}}{\partial x \partial v}\left(\tau, x_{i}, v_{j}\right) \\
=\frac{\widetilde{P}_{i+1, j+1}(\tau)+\widetilde{P}_{i-1, j-1}(\tau)-\widetilde{P}_{i+1, j-1}(\tau)-\widetilde{P}_{i-1, j+1}(\tau)}{4 \Delta x \Delta v} .
\end{gathered}
$$

Through a long derivation, we obtain from (10) the following set of discrete equations and inequalities in matrix form:

$$
\begin{gathered}
\frac{\partial \widetilde{\mathbf{P}}}{\partial \tau}-\mathbf{C} \widetilde{\mathbf{P}} \geq 0, \quad \widetilde{\mathbf{P}} \geq \mathbf{g}, \\
\left(\frac{\partial \widetilde{\mathbf{P}}}{\partial \tau}-\mathbf{C} \widetilde{\mathbf{P}}\right)^{T}(\widetilde{\mathbf{P}}-\mathbf{g})=0,
\end{gathered}
$$


where $\widetilde{\mathbf{P}}=\left(\begin{array}{llllllllll}\widetilde{P}_{00} & \widetilde{P}_{10} & \widetilde{P}_{20} & \cdots & \widetilde{P}_{m 0} & \widetilde{P}_{01} & \widetilde{P}_{11} & \widetilde{P}_{21} & \cdots & \widetilde{P}_{m 1}\end{array}\right.$

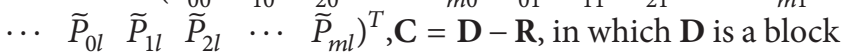
tridiagonal matrix due to the first six terms in (10), and $\mathbf{R}$ is a full matrix contributed from the two integral terms of (10). The matrix $\mathbf{D}$ can be expressed in the form

$$
\begin{gathered}
\mathbf{D}=\left(\begin{array}{cccccccc}
B_{1} & D_{1} & E_{1} & 0 & \ldots & 0 & 0 & 0 \\
0 & B_{2} & D_{2} & E_{2} & \ldots & 0 & 0 & 0 \\
\vdots & \vdots & \vdots & \vdots & \ddots & \vdots & \vdots & \vdots \\
0 & 0 & 0 & 0 & \ldots & B_{l-1} & D_{l-1} & E_{l-1} \\
0 & 0 & 0 & 0 & \ldots & 0 & \bar{B}_{l} & D_{l}
\end{array}\right), \\
\mathbf{B}_{j}=\left(\begin{array}{cccccccc}
a_{1, j} & b_{1, j} & -a_{1, j} & 0 & \ldots & 0 & 0 & 0 \\
0 & a_{2, j} & b_{2, j} & -a_{2, j} & \ldots & 0 & 0 & 0 \\
\vdots & \vdots & \vdots & \vdots & \ddots & \vdots & \vdots & \vdots \\
0 & 0 & 0 & 0 & \ldots & a_{m-1, j} & b_{m-1, j} & -a_{m-1, j} \\
0 & 0 & 0 & 0 & \ldots & 0 & 0 & b_{m, j}
\end{array}\right), \\
\mathbf{D}_{j}=\left(\begin{array}{cccccccc}
c_{1, j} & d_{1, j} & -c_{1, j} & 0 & \ldots & 0 & 0 & 0 \\
0 & c_{2, j} & d_{2, j} & -c_{2, j} & \ldots & 0 & 0 & 0 \\
\vdots & \vdots & \vdots & \vdots & \ddots & \vdots & \vdots & \vdots \\
0 & 0 & 0 & 0 & \ldots & c_{m-1, j} & d_{m-1, j} & -c_{m-1, j} \\
0 & 0 & 0 & 0 & \ldots & 0 & 0 & d_{m, j}
\end{array}\right), \\
\mathbf{E}_{j}=\left(\begin{array}{cccccccc}
a_{1, j} & -b_{1, j} & a_{1, j} & 0 & \ldots & 0 & 0 & 0 \\
0 & a_{2, j} & -b_{2, j} & a_{2, j} & \ldots & 0 & 0 & 0 \\
\vdots & \vdots & \vdots & \vdots & \ddots & \vdots & \vdots & \vdots \\
0 & 0 & 0 & 0 & \ldots & a_{m-1, j} & -b_{m-1, j} & a_{m-1, j} \\
0 & 0 & 0 & 0 & \ldots & 0 & 2 a_{m, j} & -b_{m, j}
\end{array}\right),
\end{gathered}
$$

in which $a_{i j}=-i j \sigma \rho / 4, b_{i j}=\left(\alpha(\varphi-j \Delta v)-\rho \sigma \theta_{t} j \Delta v-\right.$ $\left.j \sigma^{2}\right) / 2 \Delta v, c_{i j}=\left(i\left(r-k_{1}+\theta_{t} k_{2}\right)-j \Delta v i^{2}\right) / 2$, and $d_{i j}=$ $r+k_{0}-\theta_{t} k_{1}+j \Delta v i^{2}+\left(j \sigma^{2} / \Delta v\right)$.

To calculate the matrix $\mathbf{R}$ due to the two integral terms in (10), let $z=X y$ and rewrite the integrals by

$$
\begin{aligned}
I_{1}(\tau, X, V) & =\int_{0}^{\infty} \widetilde{P}(\tau, X y, V) f(y) d y \\
& =\int_{0}^{\infty} \frac{\widetilde{P}(\tau, z, V) f(z / X)}{X d z}, \\
I_{2}(\tau, X, V) & =\int_{0}^{\infty} \widetilde{P}(\tau, X y, V)(y-1) f(y) d y \\
& =\int_{0}^{\infty} \frac{\widetilde{P}(\tau, z, V)(z / X-1) f(z / X)}{X d z} .
\end{aligned}
$$

The above integrals can then be discretized by using linear interpolation, namely,

$$
I_{1(i, j)}^{\tau}=\int_{0}^{\infty} \frac{\widetilde{P}(\tau, z, j \Delta v) f(z / i \Delta x)}{(i \Delta x)} d z \approx \sum_{k=0}^{m-1} A_{i, j}^{\tau, k},
$$

where

$$
\begin{aligned}
& A_{i, j}^{\tau, k}= \int_{k \Delta x}^{(k+1) \Delta x} \frac{\widetilde{P}(\tau, z, j \Delta v) f(z / i \Delta x)}{(i \Delta x)} d z \\
&=\int_{k \Delta x}^{(k+1) \Delta x}\left[\frac{(k+1) \Delta x-z}{\Delta x} \widetilde{P}(\tau, k \Delta x, j \Delta v)\right. \\
&\left.+\frac{z-k \Delta x}{\Delta x} \widetilde{P}(\tau,(k+1) \Delta x, j \Delta v)\right] \\
& \times \frac{f(z / i \Delta x)}{(i \Delta x)} d z \\
&=\frac{1}{2}[k \widetilde{P}(\tau,(k+1) \Delta x, j \Delta v) \\
&-(k+1) \widetilde{P}(\tau, k \Delta x, j \Delta v)] \\
& \times\left[\operatorname{erf}\left(\frac{\mu-\ln ((k+1) / i)}{\sqrt{2} \sigma}\right)\right. \\
&\left.\quad-\operatorname{erf}\left(\frac{\mu-\ln (k / i)}{\sqrt{2} \sigma}\right)\right] \\
&+\frac{1}{2} i \exp \left(\frac{\sigma^{2}}{2}+\mu\right) \\
& \times[\widetilde{P}(\tau, k \Delta x, j \Delta v)-\widetilde{P}(\tau,(k+1) \Delta x, j \Delta v)] \\
& \times\left[\operatorname{erf}\left(\frac{\mu-\ln ((k+1) / i)+\sigma^{2}}{\sqrt{2} \sigma}\right)\right. \\
&\left.\quad-\operatorname{erf}\left(\frac{\mu-\ln (k / i)+\sigma^{2}}{\sqrt{2} \sigma}\right)\right] .
\end{aligned}
$$

Let $e_{1 i}^{k}=(1 / 2)(\operatorname{erf}((\mu-\ln ((k+1) / i)) / \sqrt{2} \sigma)-\operatorname{erf}((\mu-$ $\ln (k / i)) / \sqrt{2} \sigma))$, and $e_{2 i}^{k}=(1 / 2) i \exp \left(\sigma^{2} / 2+\mu\right)(\operatorname{erf}((\mu-\ln ((k+$ 1)/i) $\left.\left.\left.+\sigma^{2}\right) / \sqrt{2} \sigma\right)-\operatorname{erf}\left(\left(\mu-\ln (k / i)+\sigma^{2}\right) / \sqrt{2} \sigma\right)\right)$. Then (19) becomes

$$
\begin{aligned}
A_{i, j}^{\tau, k}= & \left(e_{2 i}^{k}-(k+1) e_{1 i}^{k}\right) \widetilde{P}(\tau, k \Delta x, j \Delta v) \\
& +\left(k e_{1 i}^{k}-e_{2 i}^{k}\right) \widetilde{P}(\tau,(k+1) \Delta x, j \Delta v), \\
I_{1(i, j)}^{\tau} \approx & \sum_{k=0}^{m-1} A_{i, j}^{\tau, k}=e_{2, i}^{0}-2 e_{1, i}^{0} \\
& +\sum_{k=1}^{m-1}\left[(k-1) e_{1, i}^{k-1}-e_{2, i}^{k-1}+e_{2, i}^{k}-(k+1) e_{1, i}^{k}\right] \\
& +(m-1) e_{1, i}^{m-1}-e_{2, i}^{m-1},
\end{aligned}
$$


where

$$
\begin{gathered}
\mathbf{A}=\left(\begin{array}{cccc}
A_{0} & 0 & \ldots & 0 \\
0 & A_{0} & \ldots & 0 \\
\vdots & \vdots & \ddots & \vdots \\
0 & 0 & \ldots & A_{0}
\end{array}\right), \\
\mathbf{A}_{0}=\left(\begin{array}{cccccc}
a_{0,0} & a_{0,1} & a_{0,2} & \ldots & a_{0, m-1} & a_{0, m} \\
a_{1,0} & a_{1,1} & a_{1,2} & \ldots & a_{1, m-1} & a_{1, m} \\
a_{2,0} & a_{2,1} & a_{2,2} & \ldots & a_{2, m-1} & a_{2, m} \\
\vdots & \vdots & \vdots & \vdots & \vdots & \vdots \\
a_{m, 0} & a_{m, 1} & a_{m, 2} & \ldots & a_{m, m-1} & a_{m, m}
\end{array}\right)
\end{gathered}
$$

in which $a_{i, 0}=e_{2, i}^{0}-e_{1, i}^{0}, a_{i, k}=(k-1) e_{1, i}^{k-1}-e_{2, i}^{k-1}+e_{2, i}^{k}-(k+$ 1) $e_{1, i}^{k}, k=1,2, \ldots, m-1$, and $a_{i, m}=(m-1) e_{1, i}^{m-1}-e_{2, i}^{m-1}, i=$ $0,1, \ldots, m$. Hence $I_{1}^{\tau}=\mathbf{A} \widetilde{\mathbf{P}}$.

In a similar way, we obtain $I_{2}^{\tau}=\mathbf{B} \widetilde{\mathbf{P}}$, where

$$
\begin{gathered}
\mathbf{B}=\left(\begin{array}{cccc}
B_{0} & 0 & \ldots & 0 \\
0 & B_{0} & \ldots & 0 \\
\vdots & \vdots & \ddots & \vdots \\
0 & 0 & \ldots & B_{0}
\end{array}\right), \\
\mathbf{B}_{0}=\left(\begin{array}{cccccc}
b_{00} & b_{01} & b_{02} & \ldots & b_{0, m-1} & b_{1, m} \\
b_{10} & b_{11} & b_{12} & \ldots & b_{1, m-1} & b_{1, m} \\
b_{20} & b_{21} & b_{22} & \ldots & b_{2, m-1} & b_{2, m} \\
\vdots & \vdots & \vdots & \vdots & \vdots & \vdots \\
b_{m 0} & b_{m 1} & b_{m 2} & \ldots & b_{m, m-1} & b_{m, m}
\end{array}\right)
\end{gathered}
$$

in which $b_{i 0}=e_{4 i}^{0}-2 e_{3 i}^{0}-e_{2 i}^{0}-2 e_{1 i}^{0}, b_{i, k}=(k-1) e_{3, i}^{k-1}-e_{4, i}^{k-1}+$ $e_{4, i}^{k}-(k+1) e_{3, i}^{k}-(k-1) e_{1, i}^{k-1}+e_{2, i}^{k-1}-e_{2, i}^{k}+(k+1) e_{1, i}^{k}, k=$ $1,2, \ldots, m-1, b_{i m}=(m-1) e_{3, i}^{m-1}-e_{4, i}^{m-1}-(m-1) e_{1, i}^{m-1}+$ $e_{2, i}^{m-1}, i=0,1, \ldots, m, e_{3 i}^{k}=(1 / 2) \exp \left(\sigma^{2} / 2+\mu\right) \times[\operatorname{erf}((\mu-$ $\left.\left.\left.\ln ((k+1) / i)+\sigma^{2}\right) / \sqrt{2} \sigma\right)-\operatorname{erf}\left(\left(\mu-\ln (k / i)+\sigma^{2}\right) / \sqrt{2} \sigma\right)\right]$, and $e_{4 i}^{k}=\left(1 / 2 i \sqrt{1-2 \sigma^{2}}\right) \exp \left(\mu^{2} /\left(1-2 \sigma^{2}\right)\right) \times\left[\operatorname{erf}\left(\left(\sqrt{1-2 \sigma^{2}}(\mu-\right.\right.\right.$ $\left.\ln ((k+1) / i))) / \sqrt{2} \sigma-\sqrt{2} \mu \sigma / \sqrt{1-2 \sigma^{2}}\right)-\operatorname{erf}\left(\left(\sqrt{1-2 \sigma^{2}}(\mu-\right.\right.$ $\left.\left.\ln (k / i))) / \sqrt{2} \sigma-\sqrt{2} \mu \sigma / \sqrt{1-2 \sigma^{2}}\right)\right]$.

Hence, the integral part of the operator $L$ is $\mathbf{R}=\left(\mathbf{A}-\theta_{\tau} \mathbf{B}\right)$.

Now for American options we obtain a semidiscrete LCP. The Crank-Nicolson method [15] is then used for time discretization. Let $\Delta \tau=\Gamma / n$; we obtain

$$
\begin{gathered}
\left(\mathbf{I}-\frac{1}{2} \Delta \tau \mathbf{C}^{(n)}\right) \widetilde{\mathbf{P}}^{(n)} \geq\left(\mathbf{I}+\frac{1}{2} \Delta \tau \mathbf{C}^{(n+1)}\right) \widetilde{\mathbf{P}}^{(n+1)}, \\
\widetilde{\mathbf{P}}^{(n+1)}+\widetilde{\mathbf{P}}^{(n)} \geq 2 \mathbf{g} \\
\left(\left(\mathbf{I}-\frac{1}{2} \Delta \tau \mathbf{C}^{(n)}\right) \widetilde{\mathbf{P}}^{(n)}-\left(\mathbf{I}+\frac{1}{2} \Delta \tau \mathbf{C}^{(n+1)}\right) \widetilde{\mathbf{P}}^{(n+1)}\right)^{T} \\
\times\left(\widetilde{\mathbf{P}}^{(n+1)}+\widetilde{\mathbf{P}}^{(n)}-2 \mathbf{g}\right)=0
\end{gathered}
$$

TABLE 1: The basic parameter values used in numerical investigation.

\begin{tabular}{lc}
\hline$\mu$ & 0.23 \\
$\kappa$ & 3.46 \\
$\varphi$ & $0.0894^{2}$ \\
$\delta$ & 0.0001 \\
$r$ & 0.039 \\
$\sigma$ & 0.14 \\
$\lambda$ & 0.77 \\
$\gamma$ & -0.086 \\
$\rho$ & -0.82 \\
$T$ & 1 \\
$S_{\max }$ & 200 \\
$\nu_{\max }$ & 1 \\
$K$ & 100 \\
$N$ & 3000 \\
$I$ & 30 \\
$J$ & 30 \\
\hline
\end{tabular}

which can be denoted by

$$
\begin{array}{ll}
\operatorname{LCP}\left(\mathbf{M}, \widetilde{\mathbf{P}}^{(n+1)}, \mathbf{N} \widetilde{\mathbf{P}}^{(n)}, \mathbf{g}\right): \quad & \mathbf{N} \widetilde{\mathbf{P}}^{(n)} \geq \mathbf{M} \widetilde{\mathbf{P}}^{(n+1)}, \\
& \widetilde{\mathbf{P}}^{(n+1)}+\widetilde{\mathbf{P}}^{(n)} \geq 2 \mathbf{g}, \\
& \left(\mathbf{N} \widetilde{\mathbf{P}}^{(n)}-\mathbf{M} \widetilde{\mathbf{P}}^{(n+1)}\right)^{T} \\
& \times\left(\widetilde{\mathbf{P}}^{(n+1)}+\widetilde{\mathbf{P}}^{(n)}-2 \mathbf{g}\right)=0,
\end{array}
$$

where $\mathbf{M}=\mathbf{I}+(1 / 2) \Delta \tau \mathbf{C}^{(n+1)}$ and $\mathbf{N}=\mathbf{I}-(1 / 2) \Delta \tau \mathbf{C}^{(n)}$

The projected SOR algorithm PSOR $\left(\mathbf{M}, \widetilde{\mathbf{P}}^{(n+1)}, \mathbf{N} \tilde{\widetilde{\mathbf{P}}}^{(n)}, \mathbf{g}\right)$ can then be used to solve the LCP problem. The algorithm is as follows.

For $n=1, \operatorname{dim}(\mathbf{M})$,

$$
\begin{gathered}
\mathbf{r}_{n}=\mathbf{N} \widetilde{\mathbf{P}}_{n}-\sum_{j} \mathbf{M}_{i, j} \widetilde{\mathbf{P}}_{j}, \\
\widetilde{\mathbf{P}}_{n}=\max \left\{\widetilde{\mathbf{P}}_{j}+\frac{\omega \mathbf{r}_{n}}{\mathbf{M}_{i, j}}, \mathbf{g}_{n}\right\},
\end{gathered}
$$

where the relaxation parameter $\omega=2 /\left(1+\sqrt{1-\varphi^{2}}\right)$, wherein $\varphi=\max _{i}\left\{\left(1 / \mathbf{M}_{i, i}\right) \sum_{j \neq i}\left|\mathbf{M}_{i, j}\right|\right\}$.

\section{Numerical Results and Discussion}

In this section, we present a set of results to demonstrate the feature of the model and the robustness of the model for the determination of American put option price and then investigate the influence of market volatility. The basic parameter values used in the computation are given in Table 1. The numerical algorithm is implemented in computer by using the MATLAB 2012 software package. Figure 1 shows the payoff as the function of stock price. The numerical solutions obtained by solving the LCP problem (24) are showed in 


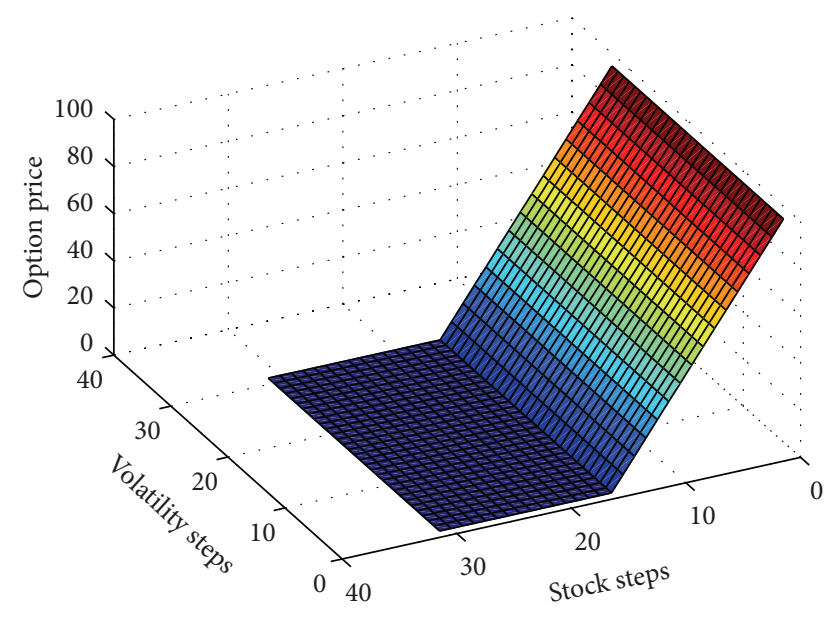

FIGURE 1: The payoff as a function of stock price (stock price $=$ stock step $* 20 / 3$; volatility $=$ volatility step $* 1 / 30$ ).

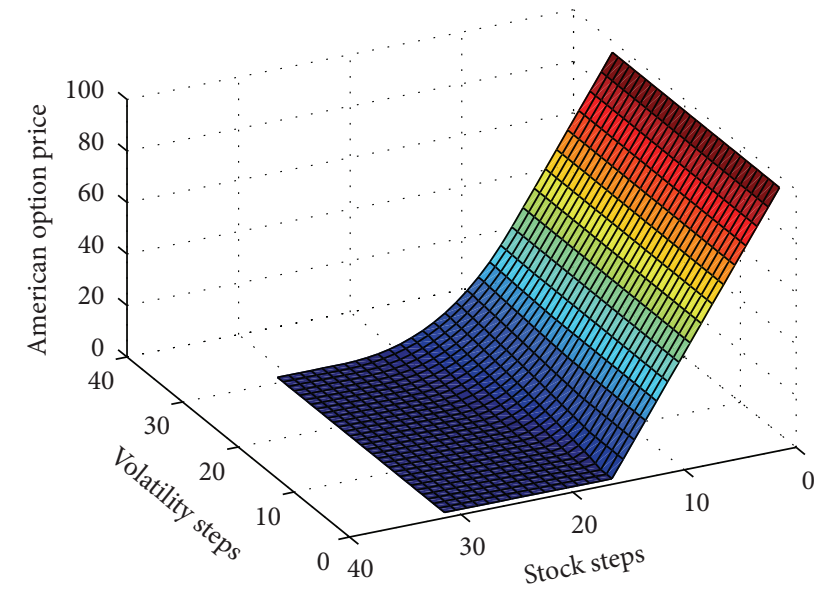

FIgURE 2: The American option price.

Figure 2. We also compute the value of American option price under various values of volatility. The results are plotted in Figure 3.

From the numerical results, we can see that volatility has no influence on the payoff of American option, but it plays an important role in American option pricing. From Figures 2 and 3 , we can see that, when the stock price is relatively low, the influence of volatility is not significant, while, as the stock price increases, the influence of volatility becomes more and more significant. It can also be found that, at the same level of stock price, the larger the volatility is, the more the influence it plays on American option price is, which is in agreement with the market reality. Thus, the results show that the model and numerical scheme are robust in capturing the feature of incomplete finance market, particularly the influence of market volatility on the price of American options.

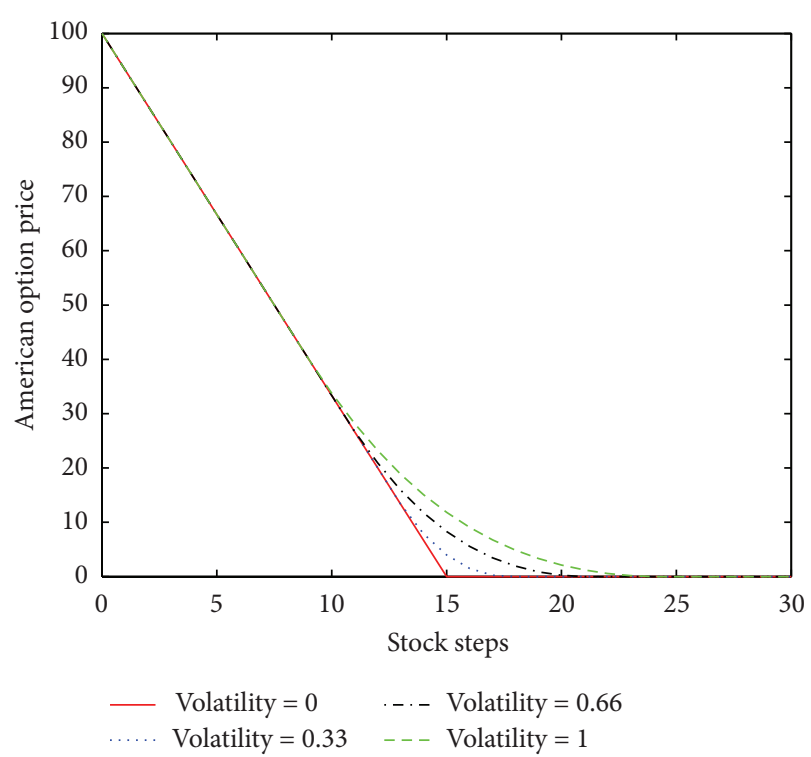

FIGURE 3: American option price under different volatilities.

\section{Appendices}

\section{A. Derivation of the Radon-Nikodym Derivative under the Minimal Martingale Measure $q$}

This appendix derives the Radon-Nikodym derivative under the minimal martingale measure $q$ and then consequently obtains (5) for American option pricing model.

According to the Doob-Meyer decomposition, the discounted risky asset price process, $\widehat{X}_{t}=e^{-\int_{0}^{t} r_{s} d s} X_{t}$, is a special semimartingale and can be written as

$$
\widetilde{X}_{t}=\widetilde{X}_{0}+M_{t}+A_{t}
$$

with

$$
\begin{gathered}
M_{t}=\int_{0}^{t} \widetilde{X}_{u} \sqrt{V_{u}} d W_{u}^{1}+\int_{0}^{t} \int_{R} \widetilde{X}_{u^{-}}(y-1) \widetilde{N}(d u, d y), \\
A_{t}=\int_{0}^{t} \widetilde{X}_{u}\left(\mu_{u}-r_{u}\right) d u,
\end{gathered}
$$

where $M_{t}$ is the martingale part of $\widetilde{X}_{t}$ and $A_{t}$ is the predictable process of finite variation.

The minimal martingale measure $q$ is unique [10] if there exists a predictable process $\beta_{t}$ that satisfies

$$
Z_{t}=\frac{d q}{d p}=1+\int_{0}^{t} \beta_{u} d M_{u} .
$$

By using (A.4), we get the theorem below for calculating the Radon-Nikodym derivative. 
Theorem 1. The Radon-Nikodym derivative under the minimal martingale measure $q$ is

$$
\begin{aligned}
Z_{t}=\exp ( & -\int_{0}^{t} \theta_{u} \sqrt{V_{u}} d W_{u}^{1}-\frac{1}{2} \int_{0}^{t} \theta_{u}^{2} V_{u} d u \\
& +\int_{0}^{t} \int_{R} \ln \left(1-\theta_{u}(y-1)\right) N(d u, d y) \\
& \left.+\int_{0}^{t} \int_{R} \theta_{u}(y-1) v(d y) d u\right) .
\end{aligned}
$$

Proof. According to the Girsanov transformation theory, the predictable process of bounded variation can be computed in terms of $Z_{t}$,

$$
-d A_{t}=\frac{d\langle M, Z\rangle_{t}}{Z_{t^{-}}},
$$

where $\langle M, Z\rangle_{t}$ is the quadratic variation process between $M$ and $Z$. Under the minimal martingale measure $q$, the predicable process of bounded variation in the Doob-Meyer decomposition of $M$ is given by

$$
\frac{d\langle M, Z\rangle_{t}}{Z_{t^{-}}}=\beta_{t} \frac{d\langle M\rangle_{t}}{Z_{t^{-}}}=-d A_{t} .
$$

Using (A.4) and (A.7), we have

$$
Z_{t}=1-\int_{0}^{t} Z_{u^{-}} \frac{d A_{u}}{d\langle M\rangle_{u}} d M_{u^{\prime}}
$$

From (A.2), we get

$$
\begin{aligned}
\langle M\rangle_{t} & =\left\langle\int_{0}^{t} \widetilde{X}_{u} \sqrt{V_{u}} d W_{u}^{1}+\int_{0}^{t} \int_{R} \widetilde{X}_{u^{-}} y \widetilde{N}(d u, d y)\right\rangle \\
& =\int_{0}^{t} \widetilde{X}_{u}^{2}\left(\sqrt{V_{u}}\right)^{2} d u+\int_{0}^{t} \int_{R} \widetilde{X}_{u^{-}}^{2}(y-1)^{2} v(d y) d u \\
& =\int_{0}^{t} \widetilde{X}_{u}^{2}\left(V_{u}+\int_{R}(y-1)^{2} v(d y)\right) d u .
\end{aligned}
$$

Let $d Y_{u}=-\left(d A_{u} / d\langle M\rangle_{u}\right) d M_{u}$; then (A.8) can be written as $Z_{t}=1+\int_{0}^{t} Z_{u^{-}} d Y_{u}$. Hence,

$$
\begin{aligned}
d Y_{u}= & \frac{\widetilde{X}_{u}\left(\mu_{u}-r_{u}\right) d u}{\widetilde{X}_{u}^{2}\left(V_{u}+\int_{R}(y-1)^{2} v(d y)\right) d u} \\
& \times\left(\widetilde{X}_{u} \sqrt{V_{u}} d W_{u}^{1}+\int_{R} \widetilde{X}_{u^{-}}(y-1) \widetilde{N}(d u, d y)\right) \\
= & \frac{\left(\mu_{u}-r_{u}\right)\left(\sqrt{V_{u}} d W_{u}^{1}+\int_{R}(y-1) \widetilde{N}(d u, d y)\right)}{V_{u}+\int_{R}(y-1)^{2} v(d y)} .
\end{aligned}
$$

Because $Z_{t}$ is the Doléans-Dade exponential, we obtain

$$
\begin{gathered}
Z_{t}=1+\int_{0}^{t} Z_{u^{-}} d Y_{u}, \quad Z_{0}=1, \\
d Y_{u}=-\theta_{u}\left(\sqrt{V_{u}} d W_{u}^{1}+\int_{R}(y-1) \widetilde{N}(d u, d y)\right),
\end{gathered}
$$

where

$$
\theta_{u}=\frac{\mu_{u}-r_{u}}{V_{u}+\int_{R}(y-1)^{2} v(d y)} .
$$

Solving equations (A.11), we obtain $Z_{t}$ as given in Theorem 2 .

\section{B. Derivation of the Partial Integrodifferential Equation (7)}

Here we derive the partial integrodifferential equation (PIDE) (7), which is used in Section 2 to construct the linear complementarity problem (LCP) for the pricing of American put option.

Under the measure of minimal martingale $q$, the price of American put option $P\left(t, X_{t}, V_{t}\right)$, having strike price $K$ and maturity date $T$, is as follows:

$$
P\left(t, X_{t}, V_{t}\right)=\max _{\tau} \mathbf{E}^{q}\left[e^{-\int_{t}^{\tau} r_{u} d u}\left(K-X_{\tau}\right)^{+} \mid F_{t}\right],
$$

where $\tau$, representing the stopping times in $[0, T]$, is to be determined to maximize the discounted option payoff.

To derive the LCP problem for the American put option, we first need to derive the following theorem for fixed $\tau$, which is for European put option.

Theorem 2. The price of the European option satisfies the following PIDE:

$$
\begin{aligned}
& -r_{t} P\left(t, X_{t}, V_{t}\right)+\frac{\partial P}{\partial t}+\frac{\partial P}{\partial X} r_{t} X_{t^{-}}+\frac{\partial P}{\partial V} \\
& \times\left(\alpha\left(\varphi-V_{t}\right)-\rho \sigma \theta_{t} V_{t}\right) \\
& +\frac{1}{2} \frac{\partial^{2} P}{\partial X^{2}} V_{t} X_{t}^{2}+\frac{\partial^{2} P}{\partial X \partial V} X_{t} V_{t} \sigma \rho+\frac{1}{2} \frac{\partial^{2} P}{\partial X^{2}} V_{t} X_{t}^{2} \\
& +\int_{R}\left(P\left(t, X_{t} y, V_{t}\right)-P\left(t, X_{t}, V_{t}\right)-(y-1) \frac{\partial P}{\partial X} X_{t^{-}}\right) \\
& \times v(d y)=0 .
\end{aligned}
$$

Proof. Using the Itô formula of semimartingales, we get

$$
\begin{aligned}
d\left(e^{-\int_{t}^{T} r_{u} d u} P\left(t, X_{t}, V_{t}\right)\right) \\
=-r_{t} e^{-\int_{0}^{t} r_{u} d u} P\left(t, X_{t}, V_{t}\right) d t+e^{-\int_{0}^{t} r_{u} d u} \frac{\partial P}{\partial t} d t \\
+e^{-\int_{0}^{t} r_{u} d u} \frac{\partial P}{\partial X} d X+e^{-\int_{0}^{t} r_{u} d u} \frac{\partial P}{\partial V} d V \\
+\frac{1}{2} e^{-\int_{0}^{t} r_{u} d u} \frac{\partial^{2} P}{\partial X^{2}} d X d X \\
\quad+e^{-\int_{0}^{t} r_{u} d u} \frac{\partial^{2} P}{\partial X \partial V} d X d V+\frac{1}{2} e^{-\int_{0}^{t} r_{u} d u} \frac{\partial^{2} P}{\partial V^{2}} d V d V \\
\quad+e^{-\int_{t}^{T} r_{u} d u} \int_{R}\left(P\left(t, X_{t} y, V_{t}\right)-P\left(t, X_{t}, V_{t}\right)\right) N(d t, d y)
\end{aligned}
$$


By substituting (5) into the equation above, we obtain

$$
\begin{aligned}
d\left(e^{-\int_{t}^{T} r_{u} d u} P\left(t, X_{t}, V_{t}\right)\right) & \\
=e^{-\int_{0}^{t} r_{u} d u}( & -r_{t} P\left(t, X_{t}, V_{t}\right) d t+\frac{\partial P}{\partial t} d t \\
& +\frac{\partial P}{\partial X} X_{t^{-}}\left(\mu d t+\sqrt{V_{t}} d \widetilde{W}_{t}^{1}-\theta_{t} V_{t} d t\right. \\
& -\int_{R} \theta_{t}(y-1)^{2} v(d y) d t \\
& +\frac{\partial P}{\partial V}\left(\alpha\left(\varphi-V_{t}\right) d t+\sigma \sqrt{V_{t}} d \widetilde{W}_{t}^{2}-\rho \sigma \theta_{t} V_{t} d t\right) \\
& +\frac{1}{2} \frac{\partial^{2} P}{\partial X^{2}} V X_{t^{-}}^{2} d t \\
& +\frac{\partial^{2} P}{\partial X \partial V} X V \rho \sigma d t+\frac{1}{2} \frac{\partial^{2} P}{\partial V^{2}} V \sigma^{2} d t \\
& +\int_{R}\left(P\left(t, X_{t} y, V_{t}\right)-P\left(t, X_{t}, V_{t}\right)\right) \widetilde{N}(d t, d y) \\
& \left.\left.+\int_{R}\left(t, X_{t} y, V_{t}\right)-P\left(t, X_{t}, V_{t}\right)\right) \widetilde{v}(d y) d t\right) .
\end{aligned}
$$

By combining the like terms of $d t$, we get

$$
\begin{aligned}
& d\left(e^{-\int_{t}^{T} r_{u} d u} P(t,\right.\left.\left.X_{t}, V_{t}\right)\right) \\
&=e^{-\int_{0}^{t} r_{u} d u}(-r_{t} P\left(t, X_{t}, V_{t}\right)+\frac{\partial P}{\partial t}+\frac{\partial P}{\partial X} r_{t} X_{t^{-}} \\
&+ \frac{\partial P}{\partial V}\left(\alpha\left(\varphi-V_{t}\right) \rho \sigma \theta_{t}\right)+\frac{1}{2} \frac{\partial^{2} P}{\partial X^{2}} V X_{t^{-}}^{2} \\
&+ \frac{\partial^{2} P}{\partial X \partial V} X V \rho \sigma+\frac{1}{2} \frac{\partial^{2} P}{\partial V^{2}} V \sigma^{2} \\
&+\int_{R}\left(P\left(t, X_{t} y, V_{t}\right)-P\left(t, X_{t}, V_{t}\right)\right. \\
&+e^{-\int_{0}^{t} r_{u} d u}\left(\frac{\partial P}{\partial X} X_{t^{-}} \sqrt{V_{t}} d \widetilde{W}_{t}^{1}+\frac{\partial P}{\partial V} \sigma \sqrt{V_{t}} d \widetilde{W}_{t}^{2}\right. \\
&+\int_{R}\left(P\left(t, X_{t} y, V_{t}\right)\right. \\
&\left.\left.-P\left(t, X_{t}, V_{t}\right)\right) \widetilde{N}(d t, d y)\right) .
\end{aligned}
$$

Because the discounted price of the European style option is a martingale, we set the drift term to zero, and consequently we obtain the equation as given in Theorem 2 . By rearranging and simplifying (B.2), we obtain (7) in Section 2. Consequently, based on the operator $L$ derived from this theorem, the LCP problem (11) is constructed in Section 2 for solving the American option model.

\section{Conflict of Interests}

The authors declare that there is no conflict of interests regarding the publication of this paper.

\section{References}

[1] F. Black and M. Scholes, "The pricing of options and corporate liabilities," Political Economy, vol. 9, pp. 69-107, 1973.

[2] R. C. Merton, "Theory of rational option pricing," The Rand Journal of Economics, vol. 4, pp. 141-183, 1973.

[3] S. Heston, "A closed-form solution for options with stochastic volatility with applications to bond and currency options," Review of Financial Studies, vol. 6, no. 2, pp. 327-343, 1993.

[4] R. Merton, "Option pricing when underlying stock returns are discontinuous," Journal of Financial Economics, vol. 3, no. 1, pp. 125-144, 1976.

[5] F. E. Benth and T. Meyer-Brandis, "The density process of the minimal entropy martingale measure in a stochastic volatility model with jumps," Finance and Stochastics, vol. 9, no. 4, pp. 563-575, 2005.

[6] D. Bates, "The crash of '87: was it expected? The evidence from options markets," Journal of Finance, vol. 46, no. 3, pp. 1009$1044,1991$.

[7] J. Kallsen, "A utility maximization approach to hedging in incomplete markets," Mathematical Methods of Operations Research, vol. 50, no. 2, pp. 321-338, 1999.

[8] T. Bielecki and M. Jeanblanc, Indifference Pricing of Defaultable Claims, Princeton University Press, 2004.

[9] J. Cvitanić, W. Schachermayer, and H. Wang, "Utility maximization in incomplete markets with random endowment," Finance and Stochastics, vol. 5, no. 2, pp. 259-272, 2001.

[10] H. Föllmer and M. Schweizer, "Hedging of contingent claims under incomplete information," in Applied Stochastic Analysis (London, 1989), vol. 5 of Stochastics Monographs, pp. 389-414, Gordon and Breach, New York, NY, USA, 1991.

[11] X. Ruan, W. Zhu, S. Li, and J. Huang, "Option pricing under risk-minimization criterion in an incomplete market with the finite difference method," Mathematical Problems in Engineering, vol. 2013, Article ID 165727, 9 pages, 2013.

[12] S. Ikonen and J. Toivanen, "Efficient numerical methods for pricing American options under stochastic volatility," Numerical Methods for Partial Differential Equations, vol. 24, no. 1, pp. 104-126, 2008.

[13] D. Tavella and C. Randall, Pricing Financial Instruments: The Finite Difference Method, John Wiley and Sons, Chichester, UK, 2000.

[14] S. Salmi and J. Toivanen, "An iterative method for pricing American options under jump-diffusion models," Applied Numerical Mathematics, vol. 61, no. 7, pp. 821-831, 2011.

[15] S. Ikonen and J. Toivanen, "Pricing American options using LU decomposition," Applied Mathematical Sciences, vol. 1, no. 4952, pp. 2529-2551, 2007. 


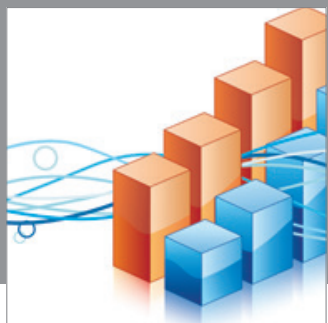

Advances in

Operations Research

mansans

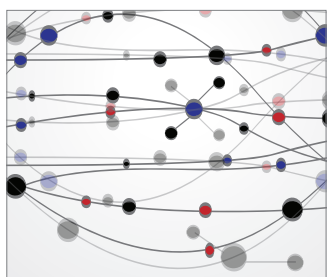

The Scientific World Journal
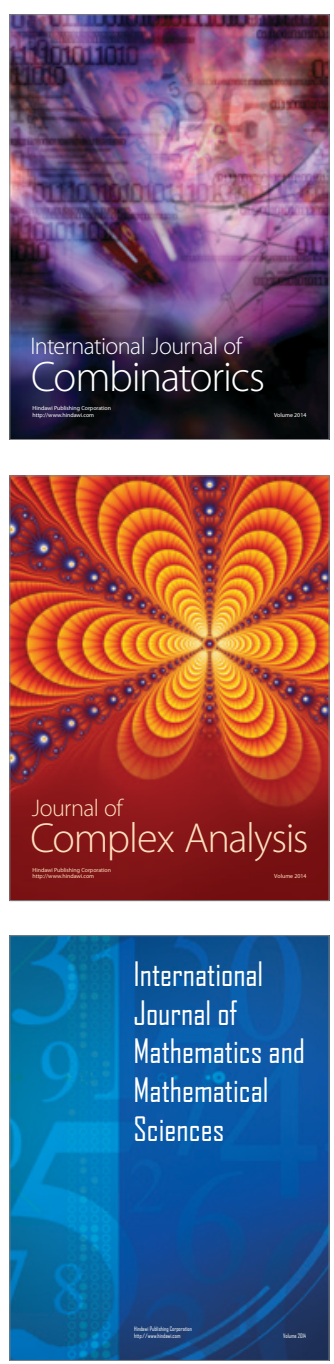
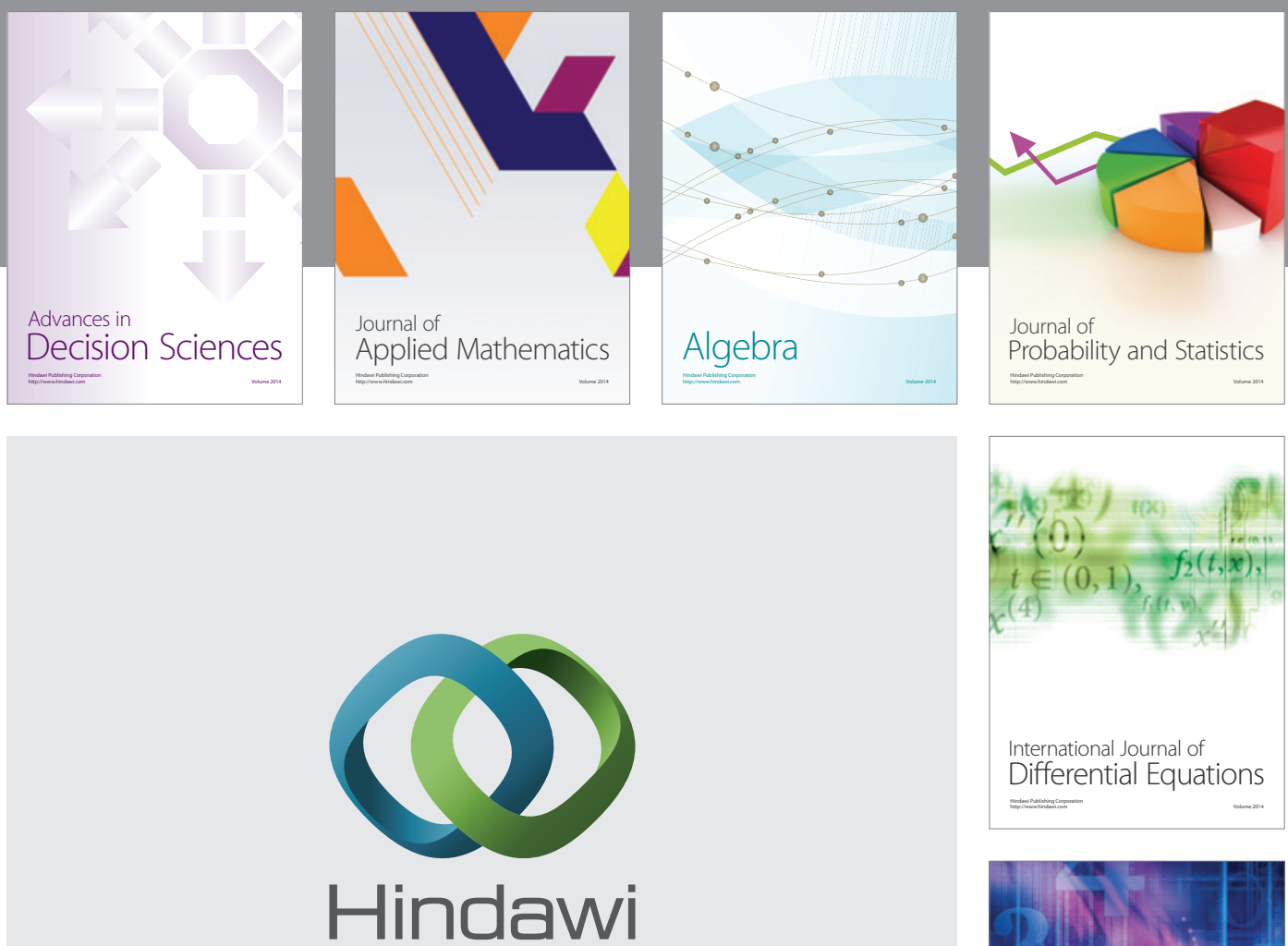

Submit your manuscripts at http://www.hindawi.com
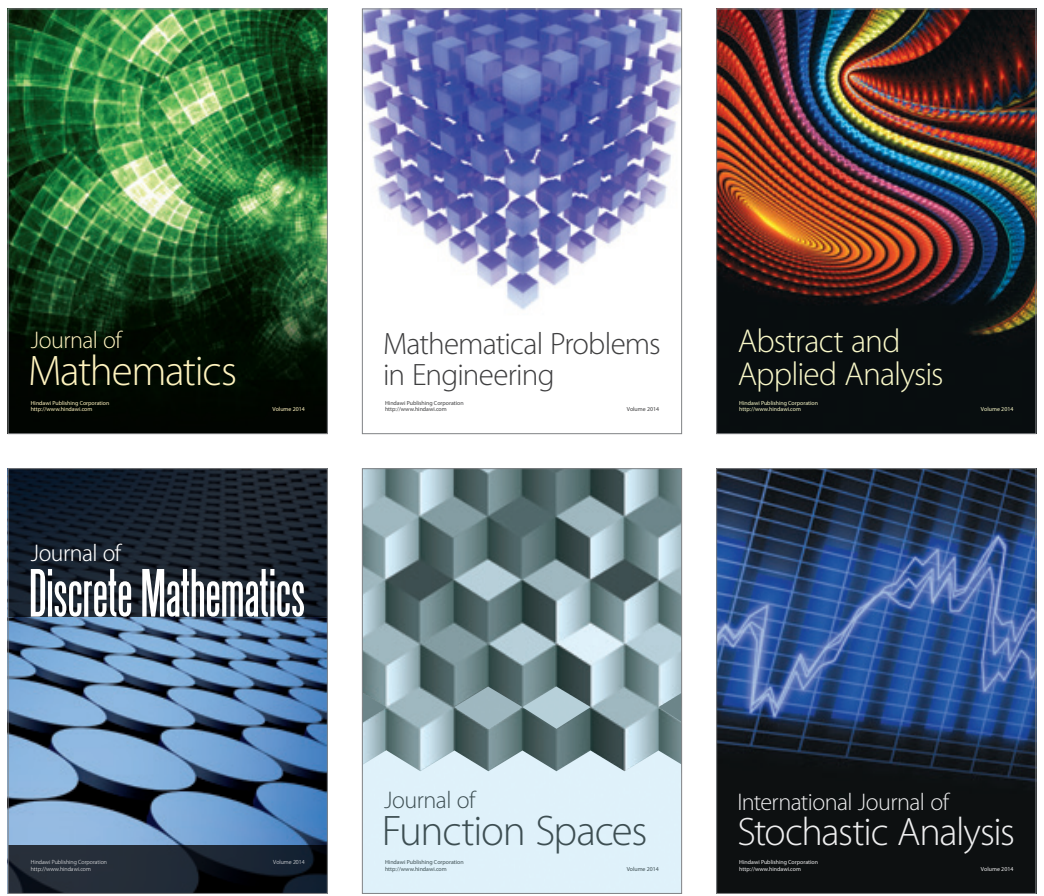

Journal of

Function Spaces

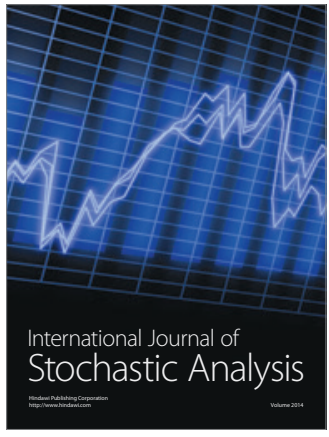

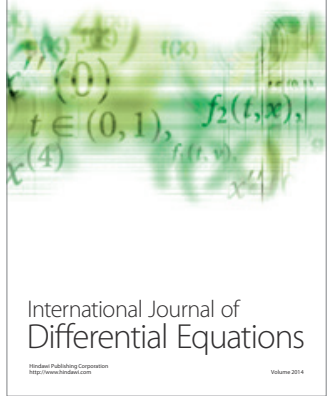
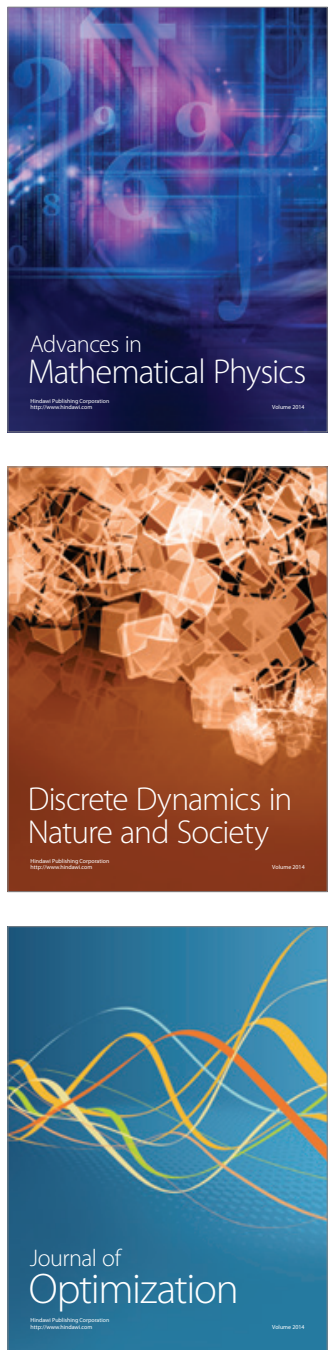